\title{
ON THE FIRST PASSAGE TIME FOR BROWNIAN MOTION SUBORDINATED BY A LÉVY PROCESS
}

\author{
T. R. HURD, ${ }^{*}$ McMaster University
}

A. KUZNETSOV, ${ }^{* *}$ York University

\begin{abstract}
In this paper we consider the class of Lévy processes that can be written as a Brownian motion time changed by an independent Lévy subordinator. Examples in this class include the variance-gamma (VG) model, the normal-inverse Gaussian model, and other processes popular in financial modeling. The question addressed is the precise relation between the standard first passage time and an alternative notion, which we call the first passage of the second kind, as suggested by Hurd (2007) and others. We are able to prove that the standard first passage time is the almost-sure limit of iterations of the first passage of the second kind. Many different problems arising in financial mathematics are posed as first passage problems, and motivated by this fact, we are led to consider the implications of the approximation scheme for fast numerical methods for computing first passage. We find that the generic form of the iteration can be competitive with other numerical techniques. In the particular case of the VG model, the scheme can be further refined to give very fast algorithms.
\end{abstract}

Keywords: Brownian motion; first passage; time change; Lévy subordinator; stopping time; financial models

2000 Mathematics Subject Classification: Primary 60J75

Secondary 60G51; 91B28

\section{Introduction}

First passage problems are a classic aspect of stochastic processes that arise in many areas of application. In mathematical finance, for example, first passage problems lie at the heart of such issues as credit risk modeling, pricing barrier options, and the optimal exercise of American options. If $X_{t}$ is any process with initial value $X_{0}=x_{0}$, the first passage time to a lower level $b$ is defined to be the stopping time

$$
t_{b}^{*}\left(x_{0}\right)=\inf \left\{t \geq 0 \mid X_{t} \leq b\right\}
$$

The distributional properties of $t^{*}$ can be easily obtained when the underlying process $X$ is a diffusion (see [7, pp. 25-27]), but, when $X$ has jumps, the situation is much more challenging.

Results on Wiener-Hopf-type factorizations (see [3, p. 336], [6, pp. 159-166], [15, pp. 145158], and [16]) have proved to be very useful for studying first passage time problems for Lévy processes. Probably the best known result of this type is the following identity (see [6, p. 165]

Received 30 May 2008; revision received 5 December 2008.

* Postal address: Department of Mathematics and Statistics, McMaster University, Hamilton, ON L8S 4K1, Canada.

Email address: hurdt@mcmaster.ca

** Postal address: Department of Mathematics and Statistics, York University, Toronto, ON M3J 1P3, Canada. 
and $[15$, p. 147]):

$$
\frac{q}{q+\psi(\lambda)}=\Phi_{q}^{+}(\lambda) \Phi_{q}^{-}(\lambda)
$$

Here $\psi(\lambda)$ is the characteristic exponent of $X_{t} ; \Phi_{q}^{+}(\lambda)$ and $\Phi_{q}^{-}(\lambda)$ are characteristic functions of infinitely divisible random variables $S_{\tau(q)}$ and $X_{\tau(q)}-S_{\tau(q)}$, where $S_{t}=\sup \left\{X_{s}: s \leq t\right\}$ is the supremum process and $\tau(q)$ is an exponential random variable with parameter $q$, independent of $X_{t}$.

We can efficiently recover functions $\Phi^{ \pm}$using (1) when $\psi(\lambda)$ is a rational function. A wellstudied class of processes for which this approach works well consists of Lévy processes with phase-type distributed jumps (see [1], [3], [4, p. 81], and [14]). Phase-type distributions are defined as the first passage time for a continuous-time, finite-state Markov chain, they form a dense class in the set of all distributions on $\mathbb{R}^{+}$, and, most importantly, if a Lévy process has phase-type jumps, its characteristic exponent is a rational function (though the converse is not true; see [19]). However, if the jumps of process $X_{t}$ are not of phase-type, we would need to approximate the jump measure of $X_{t}$ with a sequence of phase-type measures. The first problem with this approach is that there do not exist any efficient algorithms on how to achieve this. The second problem is that the degree of the polynomial equation $q+\psi(\lambda)=0$ would necessarily grow to $\infty$, which will make solving this equation very complicated. Also, see [3] and [19] for an interesting example of a distribution with rational transform which would require an infinite-degree phase-type representation.

A second general approach to first passage is to solve the Fokker-Planck equation for the probability density of $X_{t}$ conditioned on the set $\left\{t^{*}>t\right\}$. For Lévy processes, this amounts to solving a certain linear partial integral differential equation (PIDE) with nonlocal Dirichlet conditions (see [9, Proposition 12.6, Section 12.2] and [10]). In the case of Lévy processes the PIDE approach has the advantage that we can utilize the fast Fourier transform (FFT) to perform efficient computation of the convolutions involved; however, the method also involves the truncation of the state space (the real line in our case) and discretization in the $x$ and $t$ variables, and the resulting errors are not easy to control.

Our purpose here is to present a new approach to first passage problems applicable whenever the underlying Lévy process can be realized as a Lévy subordinated Brownian motion (LSBM), that is, whenever $X$ can be constructed as $\tilde{W} \circ T$, where $\tilde{W}$ is a standard drifting Brownian motion and $T$ is a nondecreasing Lévy process independent of $\tilde{W}$. The class of Lévy processes that are realizable as LSBMs is identified in [9, Theorem 4.3], and is broad enough to include many of the Lévy processes that have so far been used in finance, such as a four-parameter subclass of the Kou-Wang model, the variance gamma (VG) model, the normal-inverse Gaussian (NIG) model, and a four-parameter subclass of the generalized tempered stable process.

The basis for our approach is that, for processes that are realizable as time-changed Brownian motions, there is an alternative notion that is also relevant, namely, the first time the time change exceeds the first passage time of the Brownian motion. This notion, called the first passage of the second kind in [12], shares some characteristics with the usual first passage time and can be applied in a similar way. The usefulness of this new concept is that it can be computed efficiently in many cases where the usual first passage time cannot.

In the present paper we study the first passage for LSBMs and show how the first passage of the second kind is the first of a sequence of stopping times that converges almost surely to the first passage time. Expressed differently, first passage can be viewed as a stochastic sum of first passage times of the second kind. This sequence leads to a convergent and computable expansion for the first passage probability distribution function $p^{*}$ in terms of a similar function 
$p_{1}^{*}$ that describes the first passage distribution of the second kind. The outline of the paper is as follows. In Section 2 we define the objects needed to understand the first passage time, and we prove the expansion formula for first passage. In Section 3 we demonstrate the usefulness of this expansion by proving several explicit two-dimensional integral formulae for $p_{1}^{*}$, the first passage distribution of the second kind. In Section 4 we provide two proofs of the convergence of the expansion. The first proof is a proof of convergence in distribution and the second is a proof of convergence in the pathwise (almost-sure) sense. In Section 5 we focus on the special case of the VG model. In this important example, the formula for $p_{1}^{*}$ is reduced to a onedimensional integral (involving the exponential integral function). In Section 6, the expansion of the function $p^{*}$ is studied numerically, and found to be numerically stable and efficient.

\section{First passage for LSBMs}

Let $X_{t}$ be a general Lévy process with initial value $X_{0}=x_{0}$ and characteristics $(b, c, v)_{h}$ with respect to a truncation function $h(x)$ (see [2] or [13]). This means that $X$ is an infinitely divisible process with identical independent increments and cádlág paths (those that are continuous from the right with left limits) almost surely. Here $b, c \geq 0$ are real numbers and $v$ is a sigma-finite measure on $\mathbb{R} \backslash 0$ that integrates the function $1 \wedge x^{2}$. By the Lévy-Khintchine formula, the $\log$-characteristic function of $X_{1}$ is

$$
\log \mathrm{E}\left[\exp \left(\mathrm{i} u X_{1}\right)\right]=\mathrm{i} u b-\frac{c u^{2}}{2}+\int_{\mathbb{R} \backslash 0}\left(\mathrm{e}^{\mathrm{i} u x}-1-x h(x)\right) v(\mathrm{~d} x) .
$$

In what follows we will find it convenient to focus on the Laplace exponent of $X$ :

$$
\psi_{X}(u):=-\log \mathrm{E}\left[\exp \left(-u X_{1}\right)\right] .
$$

For simplicity of exposition, we specialize slightly by assuming that $v$ is continuous with respect to the Lebesgue measure $v(\mathrm{~d} x)=v(x) \mathrm{d} x$, and integrates $1 \wedge|x|$, allowing us to take $h(x)=0$. In this setting, the Markov generator of the process $X_{t}$ applied to any sufficiently smooth function $f(x)$ is

$$
[\mathcal{L} f](x)=b \partial_{x} f+\frac{c}{2} \partial_{x x}^{2} f+\int_{\mathbb{R} \backslash 0}(f(x+y)-f(x)) v(y) \mathrm{d} y .
$$

Definition 1. For any $b \in \mathbb{R}$, the random variable $t_{b}^{*}=t_{b}^{*}\left(x_{0}\right):=\inf \left\{t \mid X_{t} \leq b\right\}$ is called the first passage time for level $b$. When $b=0$, we drop the subscript and $t^{*}:=\inf \left\{t \mid X_{t} \leq 0\right\}$ is simply called the first passage time of $X$.

Remarks. 1. Since distributions of the increments of $X$ are invariant under time and state space shifts, we can reduce computations of $t_{b}^{*}\left(x_{0}\right)$ to computations of $t^{*}\left(x_{0}-b\right)$.

2. A general Lévy process is a mixture of a continuous Brownian motion with drift and a pure-jump process. We say that 'downward creeping' occurs if $X_{t^{*}}=0$ and does not occur if $X_{t^{*}}<0$. Under the assumption that $v$ integrates $1 \wedge|x|$, Corollaries 3 and 4 of [21] prove that there is almost surely no downward creeping if and only if the diffusive part is 0 (i.e. $c=0$ ) and the drift $b \geq 0$. In what follows we will exclude the possibility of downward creeping. In this case, $X_{t^{*}}-X_{t^{*}-} \neq 0$, so $X$ jumps across 0 , and we can define the overshoot to be $X_{t^{*}}$.

The central object of study in this paper is the joint distribution of $t^{*}$ and the overshoot $X_{t^{*}}$, in particular the joint probability density function

$$
p^{*}\left(x_{0} ; s, x_{1}\right)=\mathrm{E}_{x_{0}}\left[\delta\left(t^{*}-s\right) \delta\left(X_{t^{*}}-x_{1}\right)\right],
$$


where we invoke the Dirac delta function $\delta(\cdot)$. The marginal density of $t^{*}$ is

$$
p^{*}\left(x_{0} ; s\right)=\int_{-\infty}^{0} p^{*}\left(x_{0} ; s, x_{1}\right) \mathrm{d} x_{1} .
$$

In the introduction we noted that results on the first passage for general Lévy processes, in particular results on the functions $p^{*}$, are difficult to obtain. For this reason, we now focus on a special class of Lévy processes that can be expressed as a drifting Brownian motion subjected to a time change by an independent Lévy subordinator. Such LSBMs have been studied in [9, Section 4.4] and [12]. The general LSBM is constructed as follows.

1. For an initial value $x_{0}>0$ and drift $\beta$, let $\tilde{W}_{T}=x_{0}+W_{T}+\beta T$ be a drifting Brownian motion.

2. For a Lévy characteristic triple $(b, 0, \mu)$ with $b \geq 0$ and $\operatorname{supp}(\mu) \subset \mathbb{R}^{+}$, let the timechange process $T_{t}$ be the associated nondecreasing Lévy process (a subordinator), taken to be independent of $W$.

3. The time-changed process $X_{t}=\tilde{W}_{T_{t}}$ is defined to be an LSBM.

So constructed, it is known that $X_{t}$ is itself a Lévy process. The process $X_{t}$ will allow creeping if and only if $b>0$ : we henceforth assume for simplicity that $b=0$. Theorem 4.3 of [9] provides a characterization for Lévy processes that are LSBMs. While, unlike the class of phase-type Lévy processes, the class of LSBMs is not dense in the class of Lévy processes, their analytic properties make them a useful and flexible class. It was observed in [12] that, for any $\operatorname{LSBM} X_{t}$, we can define an alternative notion of the first passage time, which we denote here by $\tilde{t}$.

Definition 2. For any LSBM $X_{t}=\tilde{W}_{T_{t}}$, we define the first passage time of $\tilde{W}$ to be $T^{*}=$ $T^{*}\left(x_{0}\right)=\inf \left\{T: x_{0}+W_{T}+\beta T \leq 0\right\}$. Note that $T^{*}\left(x_{0}\right)=0$ when $x_{0} \leq 0$. The first passage time of the second kind of $X_{t}$ is defined as $\tilde{t}=\tilde{t}\left(x_{0}\right)=\inf \left\{t: T_{t} \geq T^{*}\left(x_{0}\right)\right\}$.

This definition of $\tilde{t}$, and its relation to $t^{*}$, is illustrated in Figure 1.
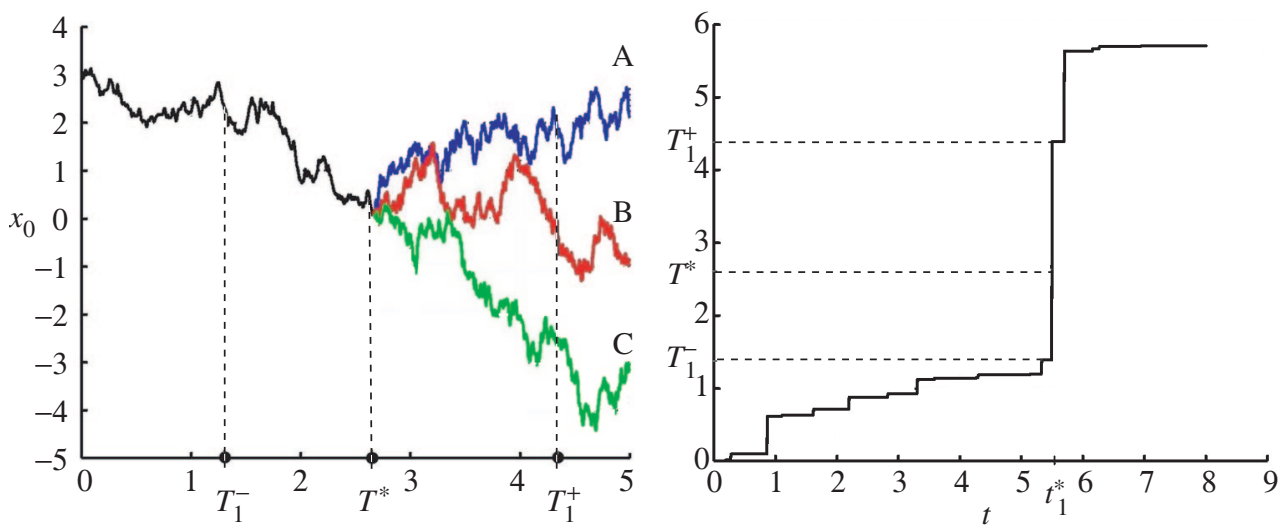

Figure 1: Three trajectories of the Brownian motion $X_{t}$ with the same $T^{*}$ and the sample path of the time change $T_{t}$ that illustrate, in general, $\tilde{t} \leq t^{*}$. On the paths B and C, $X_{t_{1}^{*}}=\tilde{W}_{T_{1}^{+}} \leq 0$, and on these paths, $t_{1}^{*}=\tilde{t}=t^{*}$. On path A, $X_{t_{1}^{*}}=\tilde{W}_{T_{1}^{+}}>0$ and so $t_{1}^{*}=\tilde{t}<t^{*}$. 
We now show that $\tilde{t}\left(x_{0}\right):=t_{1}^{*}\left(x_{0}\right)$ is the first of a sequence of approximations $\left\{t_{i}^{*}\left(x_{0}\right)\right\}_{i=1,2, \ldots}$ to the stopping time $t^{*}$. In Figure 1 we illustrate the first excursion overjump, $t_{1}^{*}$, which may be either $t^{*}$ or not. The construction of $t_{i}^{*}\left(x_{0}, \pi\right)$ is pathwise. We introduce the second argument $\pi \in \Omega$, which denotes a sample path, that is, a pair $(\omega, \tau)$, where $\omega$ is a continuous drifting Brownian path $\tilde{W}$ and $\tau$ is a cádlág sample path of the time change $T$. Thus, $\pi:(S, s) \rightarrow$ $(\omega(S), \tau(s))_{S, s \geq 0}$. The natural 'big filtration' $\left(\mathcal{F}_{t}\right)_{t \geq 0}$ for time-changed Brownian motion has

$$
\widetilde{F}_{t}=\sigma\{\omega(S), \tau(s), S \leq \tau(t), s \leq t\} .
$$

For any $t \geq 0$, there is a natural 'time translation' operation on paths $\rho_{t}:(\omega, \tau) \rightarrow\left(\omega^{\prime}, \tau^{\prime}\right)$, where $\omega^{\prime}(S)=\omega(S+\tau(t))$ and $\tau^{\prime}(s)=\tau(s+t)-\tau(t)$.

The construction of $\left\{t_{i}^{*}\left(x_{0}, \pi\right)\right\}_{i=1,2, \ldots}$ for a given sample path $\pi$ is as follows. Inductively, for $i \geq 2$, we define the time of the ith excursion overjump by

$$
t_{i}^{*}\left(x_{0}, \pi\right)=\inf \left\{t \geq t_{i-1}^{*}\left(x_{0}, \pi\right): T_{t}-T_{t_{i-1}^{*}} \geq T^{*}\left(X_{t_{i-1}^{*}}, \pi^{\prime}\right)\right\}
$$

where $\pi^{\prime}=\rho_{t_{i-1}^{*}}(\pi)$ denotes a time-shifted sample path. Note that $t_{i}^{*}\left(x_{0}, \pi\right)=t_{i-1}^{*}\left(x_{0}, \pi\right)$ if

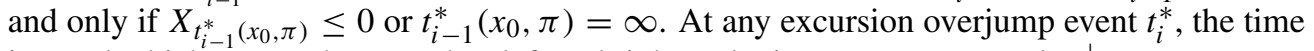
interval which covers the event has left and right endpoints $T_{i}^{-}=T_{t_{i}^{*}-}$ and $T_{i}^{+}=T_{t_{i}^{*}}$. Let

$$
p_{i}^{*}\left(x_{0} ; s, x\right)=\mathrm{E}_{x_{0}}\left[\delta\left(t_{i}^{*}-s\right) \delta\left(X_{t_{i}^{*}}-x\right)\right]
$$

denote the joint distribution of $t_{i}^{*}\left(x_{0}\right)$ and $X_{t_{i}^{*}\left(x_{0}\right)}$.

The definition of this sequence of stopping times is summarized by the pathwise equation

$$
t_{i}^{*}\left(x_{0}, \pi\right)=t_{i-1}^{*}\left(x_{0}, \pi\right) \mathbf{1}_{\left\{X_{t_{i-1}^{*}} \leq 0\right\}}+\left(t_{1}^{*}\left(X_{t_{i-1}^{*}}, \pi^{\prime}\right)+t_{i-1}^{*}\left(x_{0}, \pi\right)\right) \mathbf{1}_{\left\{X_{t_{i-1}^{*}}>0\right\}}, \quad i \geq 2,
$$

where $\pi^{\prime}=\rho_{t_{i-1}^{*}}(\pi)$. The identical increments property of the LSBM implies that the joint probability densities satisfy the recursive relation

$p_{i}^{*}\left(x_{0} ; s, x\right)=p_{1}^{*}\left(x_{0} ; s, x\right) \mathbf{1}_{\{x \leq 0\}}+\int_{0}^{\infty} \mathrm{d} y \int_{0}^{s} \mathrm{~d} u p_{1}^{*}\left(x_{0} ; u, y\right) p_{i-1}^{*}(y ; s-u, x), \quad i \geq 2$.

Similarly, the probability density function (PDF) of the first passage time $t^{*}$ satisfies the relation

$$
p_{i}^{*}\left(x_{0} ; s\right)=\int_{-\infty}^{0} p_{1}^{*}\left(x_{0} ; s, x\right) \mathrm{d} x+\int_{0}^{\infty} \mathrm{d} y \int_{0}^{s} \mathrm{~d} u p_{1}^{*}\left(x_{0} ; u, y\right) p_{i-1}^{*}(y ; s-u), \quad i \geq 2 .
$$

We note in passing that when downward creeping is included, the probability function includes an atom at $x=0$, and that when interpreted in that light, (2) and (3) are still correct.

\subsection{Examples of LSBMs}

We note here three classes of Lévy processes that can be written as LSBMs and have been used extensively in financial modeling.

1. The exponential model with parameters $(a, b, c)$ arises by taking $T_{t}$ to be the increasing process with drift $b \geq 0$ and jump measure $\mu(z)=c \mathrm{e}^{-a z}, c, a>0$, on $(0, \infty)$. The Laplace exponent of $T$ is

$$
\psi_{T}(u):=-\log \mathrm{E}\left[\exp \left(-u T_{1}\right)\right]=b u+\frac{u c}{a+u} .
$$


We can show using [9, Equation 4.14] that the resulting time-changed process $X_{t}:=\tilde{W}_{T_{t}}$ has triple $(\beta b, b, \rho)$ with

$$
\rho(y)=\frac{c}{\sqrt{\beta^{2}+2 a}} \exp \left(-\left(\sqrt{\beta^{2}+2 a}-\beta\right)(y)^{+}-\left(\sqrt{\beta^{2}+2 a}+\beta\right)(y)^{-}\right),
$$

where $(y)^{+}=\max (0, y)$ and $(y)^{-}=(-y)^{+}$. This forms a four-dimensional subclass of the six-dimensional family of exponential jump diffusions studied in [14].

2. The VG model [17] arises by taking $T_{t}$ to be a gamma process with drift defined by the characteristic triple $(b, 0, \mu)$ with $b \geq 0$ (usually, $b$ is taken to be 0 ) and jump measure $\mu(z)=(v z)^{-1} \mathrm{e}^{-z / v}, v>0$, on $(0, \infty)$. The Laplace exponent of $T_{t}, t=1$, is

$$
\psi_{T}(u):=-\log \mathrm{E}\left[\exp \left(-u T_{1}\right)\right]=b u+\frac{1}{v} \log (1+v u)
$$

The resulting time-changed process has triple $(\beta b, b, \rho)$ with

$$
\rho(y)=\frac{1}{v|y|} \exp \left(\beta x-\sqrt{\frac{2}{v}+\beta^{2}}|x|\right) .
$$

3. The NIG model with parameters $\tilde{\beta}$ and $\tilde{\gamma}$ [5] arises when $T_{t}$ is the first passage time for a second independent Brownian motion with drift $\tilde{\beta}>0$ to exceed the level $\gamma t$. Then

$$
\psi_{T}(u)=\tilde{\gamma}\left(\tilde{\beta}+\sqrt{\tilde{\beta}^{2}+2 u}\right),
$$

and the resulting time-changed process has Laplace exponent

$$
\psi_{X}(u)=x \mu+\tilde{\gamma}\left(\tilde{\beta}+\sqrt{\tilde{\beta}^{2}-u^{2}+2 \tilde{\beta} u}\right) .
$$

\section{Computing first passage of the second kind}

We have just seen that the first passage for LSBMs admits an expansion as a sum of first passage times of the second kind. In this section we show that this expansion can be useful, by proving several equivalent integral formulae for computing the structure function $p_{1}^{*}\left(x_{0} ; s, x_{1}\right)$ for general LSBMs. While the equivalence of these formulae can be demonstrated analytically, their numerical implementations will perform differently: which formula will be superior in practice is not a priori clear, but will likely depend on the range of parameters involved. For a complete picture, we provide independent proofs of the two given formulae.

Theorem 1. Let the time change $T_{t}$ have $b=0$ and Laplace exponent $\psi(u)$, and let $\tilde{W}$ have drift $\beta \neq 0$. Then

$$
\begin{aligned}
p_{1}^{*}\left(x_{0} ; s, x_{1}\right)=\frac{\exp \left(\beta\left(x_{1}-x_{0}\right)\right)}{4 \pi^{2}} \int & \int_{\mathbb{R}^{2}} \frac{\psi\left(\mathrm{i} z_{1}\right)-\psi\left(\mathrm{i} z_{2}\right)}{\mathrm{i}\left(z_{1}-z_{2}\right)} \frac{\exp \left(-s \psi\left(\mathrm{i} z_{1}\right)\right)}{\sqrt{\beta^{2}-2 \mathrm{i} z_{2}}} \\
& \times \exp \left(-x_{0} \sqrt{\beta^{2}-2 \mathrm{i} z_{1}}-\left|x_{1}\right| \sqrt{\beta^{2}-2 \mathrm{i} z_{2}}\right) \mathrm{d} z_{1} \mathrm{~d} z_{2} .
\end{aligned}
$$


Provided that the time change is not a compound Poisson process, then

$$
\begin{aligned}
p_{1}^{*}\left(x_{0} ; s, x_{1}\right)=- & \frac{2 \exp \left(\beta\left(x_{1}-x_{0}\right)\right)}{\pi^{2}} \mathrm{PV} \iint_{\left(\mathbb{R}^{+}\right)^{2}} \mathrm{~d} k_{1} \mathrm{~d} k_{2} \frac{k_{2} \cos \left|x_{1}\right| k_{1} \sin x_{0} k_{2}}{k_{1}^{2}-k_{2}^{2}} \\
& \times \exp \left(-s \psi\left(\frac{k_{1}^{2}+\beta^{2}}{2}\right)\right) \psi\left(\frac{k_{2}^{2}+\beta^{2}}{2}\right) \\
- & \begin{aligned}
-\frac{\exp \left(\beta\left(x_{1}-x_{0}\right)\right)}{\pi} \int_{\mathbb{R}^{+}} & \sin \left|x_{1}\right| k \sin x_{0} k \\
& \times \exp \left(-s \psi\left(\frac{k^{2}+\beta^{2}}{2}\right)\right) \psi\left(\frac{k^{2}+\beta^{2}}{2}\right) \mathrm{d} k .
\end{aligned}
\end{aligned}
$$

Here PV denotes that the principal value contour is taken.

Remark. The equivalence of these two formulae can be demonstrated directly by performing the change of variables $k_{j}=\mathrm{i}\left(\beta^{2}-2 \mathrm{i} z_{j}^{2}\right)^{1 / 2}, j=1,2$, followed by a deformation of the contours. Justification of the contour deformation (from the branch of a left-right symmetric hyperbola in the upper half $k_{j}$-plane to the real axis) depends on the decay of the integrand and the computation of certain residues.

\subsection{First proof of Theorem 1}

For a fixed level $h>0$, the first passage time and the overshoot of the process $T_{t}$ above the level $h$ are defined to be $\tilde{t}(h)=\inf \left\{t>0 \mid T_{t}>h\right\}$ and $\tilde{\delta}(h)=T_{\tilde{t}(h)}-h$. The PecherskiiRogozin identity [20] applied to the nondecreasing process $T$ says that

$$
\int_{0}^{\infty} \exp \left(-z_{1} h\right) \mathrm{E}\left[\exp \left(-z_{2} \tilde{\delta}(h)-z_{3} \tilde{t}(h)\right)\right] \mathrm{d} h=\frac{\psi\left(z_{1}\right)-\psi\left(z_{2}\right)}{z_{1}-z_{2}}\left(z_{3}+\psi\left(z_{1}\right)\right)^{-1} .
$$

Inversion of the Laplace transform in the above equation then leads to

$$
\mathrm{E}\left[\exp \left(-z_{2} \tilde{\delta}(h)-z_{3} \tilde{t}(h)\right)\right]=\frac{1}{2 \pi} \int_{\mathbb{R}} \frac{\psi\left(\mathrm{i} z_{1}\right)-\psi\left(z_{2}\right)}{\mathrm{i} z_{1}-z_{2}}\left(z_{3}+\psi\left(\mathrm{i} z_{1}\right)\right)^{-1} \exp \left(\mathrm{i} z_{1} h\right) \mathrm{d} z_{1} .
$$

The first passage time of the Brownian motion with drift is defined as $T^{*}=T^{*}\left(x_{0}\right)=$ $\inf \left\{T>0 \mid x_{0}+W_{T}+\beta T<0\right\}$. Next, we need to find the joint Laplace transform of $t_{1}^{*}=\inf \left\{t \mid T_{t}>T^{*}\right\}=\tilde{t}\left(T^{*}\right)$ and the overshoot $\delta^{*}=\tilde{\delta}\left(T^{*}\right)$. Since $T_{t}$ is independent of $W_{T}$, we find that

$$
\begin{aligned}
& \mathrm{E}\left[\exp \left(-z_{2} \delta^{*}-z_{3} t_{1}^{*}\right)\right] \\
& \quad=\mathrm{E}\left[\mathrm{E}\left[\exp \left(-z_{2} \tilde{\delta}\left(T^{*}\right)-z_{3} \tilde{t}\left(T^{*}\right)\right) \mid T^{*}\right]\right] \\
& \quad=\frac{1}{2 \pi} \int_{\mathbb{R}} \frac{\psi\left(\mathrm{i} z_{1}\right)-\psi\left(z_{2}\right)}{\mathrm{i} z_{1}-z_{2}}\left(z_{3}+\psi\left(\mathrm{i} z_{1}\right)\right)^{-1} \mathrm{E}\left[\exp \left(\mathrm{i} z_{1} T^{*}\right)\right] \mathrm{d} z_{1} \\
& \quad=\frac{1}{2 \pi} \int_{\mathbb{R}} \frac{\psi\left(\mathrm{i} z_{1}\right)-\psi\left(z_{2}\right)}{\mathrm{i} z_{1}-z_{2}}\left(z_{3}+\psi\left(\mathrm{i} z_{1}\right)\right)^{-1} \exp \left(-x_{0}\left(\beta+\sqrt{\beta^{2}-2 \mathrm{i} z_{1}}\right)\right) \mathrm{d} z_{1},
\end{aligned}
$$

where in the last equality we have used the following well-known result for the characteristic function of the first passage time of Brownian motion with drift:

$$
\mathrm{E}\left[\exp \left(\mathrm{i} z_{1} T^{*}\left(x_{0}\right)\right)\right]=\exp \left(-x_{0}\left(\beta+\sqrt{\beta^{2}-2 \mathrm{i} z_{1}}\right)\right) .
$$


Next we use the Fourier transform of the PDF of the Brownian motion with drift to obtain

$$
\begin{aligned}
\mathrm{E}\left[\delta\left(\tilde{W}_{t}-x_{1}\right)\right] & =\frac{\exp \left(-\left(x_{1}-\beta t\right)^{2} / 2 t\right)}{\sqrt{2 \pi t}} \\
& =\frac{\exp \left(\beta x_{1}\right)}{2 \pi} \int_{\mathbb{R}} \exp \left(-\mathrm{i} z_{2} t\right) \frac{\exp \left(-\left|x_{1}\right| \sqrt{\beta^{2}-2 \mathrm{i} z_{2}}\right)}{\sqrt{\beta^{2}-2 \mathrm{i} z_{2}}} \mathrm{~d} z_{2} .
\end{aligned}
$$

Thus, using the fact that $\tilde{W}$ is independent of $t_{1}^{*}$ and $\delta^{*}$, we obtain

$$
\begin{aligned}
& \mathrm{E}\left[\exp \left(-z_{3} t_{1}^{*}\right) \delta\left(\tilde{W}_{\delta^{*}}-x_{1}\right)\right] \\
& =\mathrm{E}\left[\mathrm{E}\left[\exp \left(-z_{3} t_{1}^{*}\right) \delta\left(\tilde{W}_{\delta^{*}}-x_{1}\right) \mid \delta^{*}\right]\right] \\
& =\frac{\exp \left(\beta x_{1}\right)}{2 \pi} \int_{\mathbb{R}} \mathrm{E}\left[\exp \left(-z_{3} t_{1}^{*}-\mathrm{i} z_{2} \delta^{*}\right)\right] \frac{\exp \left(-\left|x_{1}\right| \sqrt{\beta^{2}-2 \mathrm{i} z_{2}}\right)}{\sqrt{\beta^{2}-2 \mathrm{i} z_{2}}} \mathrm{~d} z_{2} \\
& =\frac{\exp \left(\beta\left(x_{1}-x_{0}\right)\right)}{4 \pi^{2}} \iint_{\mathbb{R}^{2}} \frac{\psi\left(\mathrm{i} z_{1}\right)-\psi\left(\mathrm{i} z_{2}\right)}{\mathrm{i}\left(z_{1}-z_{2}\right)} \frac{\left(z_{3}+\psi\left(\mathrm{i} z_{1}\right)\right)^{-1}}{\sqrt{\beta^{2}-2 \mathrm{i} z_{2}}} \\
& \quad \times \exp \left(-x_{0} \sqrt{\beta^{2}-2 \mathrm{i} z_{1}}-\left|x_{1}\right| \sqrt{\beta^{2}-2 \mathrm{i} z_{2}}\right) \mathrm{d} z_{1} \mathrm{~d} z_{2} .
\end{aligned}
$$

Now, the statement of the theorem follows after one additional Fourier inversion:

$$
\begin{aligned}
p_{1}^{*}\left(x_{0} ; s, x_{1}\right)= & \mathrm{E}\left[\delta\left(t_{1}^{*}-s\right)\left(\tilde{W}_{\delta^{*}}-x_{1}\right)\right] \\
= & \frac{1}{2 \pi} \int_{\mathbb{R}} \exp \left(\mathrm{i} z_{3} s\right) \mathrm{E}\left[\exp \left(-\mathrm{i} z_{3} t_{1}^{*}\right) \delta\left(\tilde{W}_{\delta^{*}}-x_{1}\right)\right] \mathrm{d} z_{3} \\
= & \frac{\exp \left(\beta\left(x_{1}-x_{0}\right)\right)}{4 \pi^{2}} \iint_{\mathbb{R}^{2}} \frac{\psi\left(\mathrm{i} z_{1}\right)-\psi\left(\mathrm{i} z_{2}\right)}{\mathrm{i}\left(z_{1}-z_{2}\right)} \frac{\exp \left(-s \psi\left(\mathrm{i} z_{1}\right)\right)}{\sqrt{\beta^{2}-2 \mathrm{i} z_{2}}} \\
& \quad \times \exp \left(-x_{0} \sqrt{\beta^{2}-2 \mathrm{i} z_{1}}-\left|x_{1}\right| \sqrt{\beta^{2}-2 \mathrm{i} z_{2}}\right) \mathrm{d} z_{1} \mathrm{~d} z_{2},
\end{aligned}
$$

where we have also used the Fourier integral

$$
\frac{1}{2 \pi} \int_{\mathbb{R}} \frac{\exp \left(\mathrm{i} z_{3} s\right)}{\mathrm{i} z_{3}+\psi\left(\mathrm{i} z_{1}\right)} \mathrm{d} z_{3}=\exp \left(-s \psi\left(\mathrm{i} z_{1}\right)\right) .
$$

\subsection{Second proof of Theorem 1}

The strategy of the proof is to compute

$$
I(u)=\mathrm{E}_{0, x_{0}}\left[\mathbf{1}_{\left\{s<t_{1}^{*} \leq s+u\right\}} \delta\left(X_{s+u}-x_{1}\right)\right]
$$

and then take the limit of $I(u) / \underset{\sim}{u}$ as $u \rightarrow 0+$. The key idea is to note that $X_{s+u}=X_{s-}+\tilde{W}_{T_{u}^{\prime}}^{\prime}$, where $\tilde{W}^{\prime}$ and $T^{\prime}$ are copies of $\tilde{W}$ and $T$, independent of the filtration $\mathcal{F}_{S-}$. We can then perform the above expectation via an intermediate conditioning on $\mathcal{F}_{s-}$ :

$$
\begin{aligned}
& \mathrm{E}\left[\delta\left(X_{s+u}-x_{1}\right) \mathbf{1}_{\left\{s<t_{1}^{*} \leq s+u\right\}} \mid \mathcal{F}_{s-}\right] \\
& \quad=\mathbf{1}_{\left\{s<t_{1}^{*}\right\}} \mathrm{E}\left[\delta\left(\ell+\tilde{W}_{T_{u}}^{\prime}-x_{1}\right) \mathbf{1}_{\left\{u \geq t^{\prime \prime}\right\}} \mid X_{s-}=\ell\right] .
\end{aligned}
$$

To evaluate the expectations that arise, we will need Lemma 1(ii) and (iii), below, that were stated and proved in [12]. 
Lemma 1. ([12].) (i) For any $s>0$,

$$
\begin{aligned}
& \mathrm{E}_{0, x}\left[\mathbf{1}_{\left\{s<t_{1}^{*}\right\}} \delta\left(X_{s}-y\right)\right]=\mathbf{1}_{\{y>0\}} \frac{\mathrm{e}^{\beta(y-x)}}{2 \pi} \int_{\mathbb{R}}\left(\mathrm{e}^{\mathrm{i} z(x-y)}-\mathrm{e}^{\mathrm{i} z(x+y)}\right) \\
& \times \exp \left(-s \psi\left(\frac{z^{2}+\beta^{2}}{2}\right)\right) \mathrm{d} z .
\end{aligned}
$$

(ii) For any $s>0$ and $\varepsilon \in \mathbb{R}$,

$$
\mathrm{E}_{0, x}\left[\mathbf{1}_{\left\{s \geq t_{1}^{*}\right\}} \delta\left(X_{s}-y\right)\right]=\frac{\mathrm{e}^{\beta(y-x)}}{2 \pi} \int_{\mathbb{R}+\mathrm{i} \varepsilon} \mathrm{e}^{\mathrm{i} z(x+|y|)} \exp \left(-s \psi\left(\frac{z^{2}+\beta^{2}}{2}\right)\right) \mathrm{d} z .
$$

(iii) For any $k$ in the upper half-plane,

$$
\begin{aligned}
\mathrm{E}_{0, x} & {\left[\mathbf{1}_{\left\{s<t_{1}^{*}\right\}} \exp \left(-\beta X_{s}+\mathrm{i} k X_{s}\right)\right] } \\
& =\frac{\mathrm{e}^{-\beta x}}{2 \pi} \int_{\mathbb{R}}\left(\frac{\mathrm{i}}{k-z}-\frac{\mathrm{i}}{k+z}\right) \mathrm{e}^{\mathrm{i} z x} \exp \left(-s \psi\left(\frac{z^{2}+\beta^{2}}{2}\right)\right) \mathrm{d} z .
\end{aligned}
$$

First, using (5), we find that

$$
\begin{aligned}
& \mathrm{E}\left[\delta\left(X_{s+u}-x_{1}\right) \mathbf{1}_{\left\{s<t_{1}^{*} \leq s+u\right\}} \mid X_{s-}=\ell\right] \\
& \quad=\mathbf{1}_{\left\{s<t_{1}^{*}\right\}} \frac{\exp \left(\beta\left(x_{1}-\ell\right)\right)}{2 \pi} \int_{\mathbb{R}+\mathrm{i} \varepsilon} \mathrm{d} k \exp \left(\mathrm{i} k\left(\ell+\left|x_{1}\right|\right)\right) \exp \left(-u \psi\left(\frac{k^{2}+\beta^{2}}{2}\right)\right) .
\end{aligned}
$$

When we paste this expression into the final expectation over $X_{s-}$, we can use Fubini to interchange the expectation and integral, providing that we choose $\varepsilon>0$. Then we find that

$$
I=\frac{\exp \left(\beta x_{1}\right)}{2 \pi} \int_{\mathbb{R}+\mathrm{i} \varepsilon} \mathrm{d} k \exp \left(\mathrm{i} k\left|x_{1}\right|\right) \exp \left(-u \psi\left(\frac{k^{2}+\beta^{2}}{2}\right)\right) \mathrm{E}_{0, x_{0}}\left[\exp \left(\mathrm{i} k X_{s}-\beta X_{S}\right) \mathbf{1}_{\left\{s<t_{1}^{*}\right\}}\right] .
$$

We can now use (6) to obtain

$$
\begin{aligned}
I=\frac{\exp \left(\beta\left(x_{1}-x_{0}\right)\right)}{(2 \pi)^{2}} \iint_{(\mathbb{R}+\mathrm{i} \varepsilon) \times \mathbb{R}} & \exp \left(\mathrm{i} k\left|x_{1}\right|+\mathrm{i} z x_{0}\right)\left(\frac{\mathrm{i}}{k-z}-\frac{\mathrm{i}}{k+z}\right) \\
& \times \exp \left(-u \psi\left(\frac{k^{2}+\beta^{2}}{2}\right)-s \psi\left(\frac{z^{2}+\beta^{2}}{2}\right)\right) \mathrm{d} z \mathrm{~d} k .
\end{aligned}
$$

Noting that $I(0)=0$ and taking $\lim _{u \rightarrow 0} I(u) / u$ now gives

$$
\begin{array}{rl}
p_{1}^{*}\left(x_{0} ; s, x_{1}\right)=\frac{\exp \left(\beta\left(x_{1}-x_{0}\right)\right)}{2 \pi^{2}} \iint_{(\mathbb{R}+\mathrm{i} \varepsilon) \times \mathbb{R}} & \mathrm{d} k \mathrm{~d} z \frac{\mathrm{i} z}{k^{2}-z^{2}} \exp \left(\mathrm{i} k\left|x_{1}\right|+\mathrm{i} z x_{0}\right) \\
& \times \exp \left(-s \psi\left(\frac{z^{2}+\beta^{2}}{2}\right)\right) \psi\left(\frac{k^{2}+\beta^{2}}{2}\right) .
\end{array}
$$

Here the arbitrary parameter $\varepsilon>0$ can be seen to ensure the correct prescription for dealing with the pole at $k^{2}=z^{2}$. 
Finally, the complex integration in (7) can be expressed in the following manifestly real form:

$$
\begin{array}{r}
p_{1}^{*}\left(x_{0} ; s, x_{1}\right)=-\frac{2 \exp \left(\beta\left(x_{1}-x_{0}\right)\right)}{\pi^{2}} \mathrm{PV} \iint_{\left(\mathbb{R}^{+}\right)^{2}} \mathrm{~d} k \mathrm{~d} z \frac{z \cos \left|x_{1}\right| k \sin x_{0} z}{k^{2}-z^{2}} \\
\quad \times \exp \left(-s \psi\left(\frac{k^{2}+\beta^{2}}{2}\right)\right) \psi\left(\frac{z^{2}+\beta^{2}}{2}\right) \\
-\frac{\exp \left(\beta\left(x_{1}-x_{0}\right)\right)}{\pi} \int_{\mathbb{R}^{+}} \sin \left|x_{1}\right| z \sin x_{0} z \\
\quad \times \exp \left(-s \psi\left(\frac{z^{2}+\beta^{2}}{2}\right)\right) \psi\left(\frac{z^{2}+\beta^{2}}{2}\right) \mathrm{d} z,
\end{array}
$$

involving a principal value integral plus explicit half residue terms for the poles $k= \pm z$.

\section{The iteration scheme and its convergence}

The next theorem shows that (2) can be used to compute $p^{*}\left(x_{0} ; s, x\right)$. We define a suitable $L^{\infty}$ norm for functions $f\left(x_{0} ; u, x\right)$ :

$$
\|f\|_{\infty}=\sup _{x_{0} \geq 0}\left[\int_{0}^{\infty} \int_{0}^{\infty}\left|f\left(x_{0} ; u, x\right)\right| \mathrm{d} u \mathrm{~d} x\right] .
$$

Theorem 2. The sequence $\left(p_{n}^{*}\right)_{n \geq 1}$ converges exponentially in the $L^{\infty}$ norm.

Proof. First we find from (2) that

$$
\begin{aligned}
& p_{n+1}^{*}\left(x_{0} ; s, x_{1}\right)-p_{n}^{*}\left(x_{0} ; s, x_{1}\right) \\
& \quad=\int_{0}^{\infty} \mathrm{d} y \int_{0}^{s} \mathrm{~d} u p_{1}^{*}\left(x_{0} ; s-u, y\right)\left[p_{n}^{*}\left(y ; u, x_{1}\right)-p_{n-1}^{*}\left(y ; u, x_{1}\right)\right]
\end{aligned}
$$

thus,

$$
\left\|p_{n+1}^{*}-p_{n}^{*}\right\|_{\infty} \leq C\left\|p_{n}^{*}-p_{n-1}^{*}\right\|_{\infty}
$$

where

$$
C=\sup _{x_{0} \geq 0}\left[\int_{0}^{\infty} \int_{0}^{\infty} p_{1}^{*}\left(x_{0} ; u, x\right) \mathrm{d} u \mathrm{~d} x\right] .
$$

The proof is based on the probabilistic interpretation of the constant $C$. By definition, $p_{1}^{*}\left(x_{0} ; u, x\right)$ is the joint density of $t_{1}^{*}$ and $X_{t_{1}^{*}}$; thus, we obtain

$$
C=\sup _{x_{0} \geq 0} \mathrm{P}\left(t_{1}^{*}<+\infty, X_{t_{1}^{*}}>0 \mid X_{0}=x_{0}\right)
$$

Next, using the fact that $\tilde{W}_{T^{*}}=0\left(T^{*}\right.$ is the first passage time of $X_{T}$ and $X$ is a continuous process) and the strong Markov property of the Brownian motion, we find that

$$
\begin{aligned}
C & =\mathrm{P}\left(t_{1}^{*}<+\infty, \tilde{W}_{T_{t_{1}^{*}}}-\tilde{W}_{T^{*}}>0 \mid \tilde{W}_{0}=x_{0}\right) \\
& =\mathrm{P}\left(t_{1}^{*}<+\infty, W_{\delta^{*}}+\beta \delta^{*}>0 \mid W_{0}=0\right),
\end{aligned}
$$


where the Brownian motion $W_{t}$ is independent of $T_{t}$ and $\delta^{*}=\delta^{*}\left(x_{0}\right)=T_{t_{1}^{*}}-T^{*}$ is the overshoot of the time change above $T^{*}$. Thus, we need to prove that

$$
C=\sup _{x_{0} \geq 0} \mathrm{P}\left(t_{1}^{*}<+\infty, W_{\delta^{*}}+\beta \delta^{*}>0 \mid W_{0}=0\right)<1,
$$

where $t_{1}^{*}=t_{1}^{*}\left(x_{0}\right), \delta^{*}=\delta^{*}\left(x_{0}\right)$, and the Brownian motion $W$ is independent of $t_{1}^{*}$ and $\delta^{*}$.

First we will consider the case where $\beta<0$. In this case we obtain

$$
\begin{aligned}
\mathrm{P}\left(t_{1}^{*}\right. & \left.<+\infty, W_{\Delta^{*}}+\beta \delta^{*}>0 \mid W_{0}=0\right) \\
& \leq \mathrm{P}\left(W_{\delta^{*}}+\beta \delta^{*}>0 \mid W_{0}=0\right) \\
& =\int_{0}^{\infty} \mathrm{P}\left(W_{t}+\beta t>0 \mid W_{0}=0\right) \mathrm{P}\left(\delta^{*} \in \mathrm{d} t\right) \\
& <\int_{0}^{\infty} \frac{1}{2} \mathrm{P}\left(\delta^{*} \in \mathrm{d} t\right) \\
& =\frac{1}{2},
\end{aligned}
$$

where we have used the facts that $W_{t}$ is independent of the overshoot $\delta^{*}$ and $\mathrm{P}\left(W_{t}+\beta t>\right.$ $\left.0 \mid W_{0}=0\right)<\frac{1}{2}$ for any $t$ and any $\beta<0$. Thus, in the case where the drift $\beta$ is negative we obtain an estimate $C<\frac{1}{2}$.

The case where the drift $\beta$ is positive is more complicated. We cannot use the same techniques as before, since the bound $\mathrm{P}\left(W_{t}+\beta t>0 \mid W_{0}=0\right)<\frac{1}{2}$ is no longer true: in fact, $\mathrm{P}\left(W_{t}+\beta t>\right.$ $\left.0 \mid W_{0}=0\right)$ monotonically increases to 1 as $t \rightarrow \infty$.

First we will consider the case where $x_{0}$ is bounded away from $0: x_{0} \geq c>0$. Then $x_{0}+W_{t}+\beta t$ has a positive probability of escaping to $+\infty$ and never crossing the barrier at 0 ; thus,

$$
\begin{aligned}
\mathrm{P}\left(t_{1}^{*}<+\infty, W_{\delta^{*}}+\beta \delta^{*}>0 \mid W_{0}=0\right) & \leq \mathrm{P}\left(t_{1}^{*}\left(x_{0}\right)<+\infty\right) \\
& <\mathrm{P}\left(t_{1}^{*}(c)<+\infty\right) \\
& =1-\varepsilon_{1}(c)
\end{aligned}
$$

Now we need to consider the case where $x_{0} \rightarrow 0^{+}$. The proof in this case is based on the following sequence of inequalities:

$$
\begin{aligned}
\mathrm{P}\left(t_{1}^{*}\right. & \left.<+\infty, W_{\delta^{*}}+\beta \delta^{*}>0 \mid W_{0}=0\right) \\
& \leq \mathrm{P}\left(W_{\delta^{*}}+\beta \delta^{*}>0 \mid W_{0}=0\right) \\
& =1-\mathrm{P}\left(W_{\delta^{*}}+\beta \delta^{*}<0 \mid W_{0}=0\right) \\
& =1-\int_{0}^{\infty} \mathrm{P}\left(W_{t}+\beta t<0 \mid W_{0}=0\right) \mathrm{P}\left(\delta^{*} \in \mathrm{d} t\right) \\
& <1-\int_{0}^{\tau} \mathrm{P}\left(W_{t}+\beta t<0 \mid W_{0}=0\right) \mathrm{P}\left(\delta^{*} \in \mathrm{d} t\right) \\
& <1-\int_{0}^{\tau} \mathrm{P}\left(W_{\tau}+\beta \tau<0 \mid W_{0}=0\right) \mathrm{P}\left(\delta^{*} \in \mathrm{d} t\right) \\
& =1-\mathrm{P}\left(W_{\tau}+\beta \tau<0 \mid W_{0}=0\right) \mathrm{P}\left(\delta^{*}<\tau\right),
\end{aligned}
$$

where $\tau$ is any positive number and the last inequality is true since $\mathrm{P}\left(W_{t}+\beta t<0 \mid W_{0}=0\right)$ is a decreasing function of $t$. 
Since $x_{0} \rightarrow 0^{+}$, we also have $T^{*}\left(x_{0}\right) \rightarrow 0^{+}$with probability 1 . Since $\delta^{*}$ is the overshoot of $T^{*}$, and $T^{*} \rightarrow 0^{+}$as $x_{0} \rightarrow 0^{+}$, we see that the distribution of the overshoot $\delta^{*}\left(x_{0}\right)$ converges either to the distribution of the jumps of $T_{t}$ if the time-change process $T_{t}$ is a compound Poisson process or to the Dirac delta distribution at 0 if $T_{t}$ has infinite activity of jumps. Therefore, in the case where $T_{t}$ is a compound Poisson process with the jump measure $v(\mathrm{~d} x)$ we choose $\tau$ such that $\nu([0, \tau])>0$, and if $T_{t}$ has infinite activity of jumps, we can take any $\tau>0$. Then we obtain $\lim _{x_{0} \rightarrow 0^{+}} \mathrm{P}\left(\delta^{*}\left(x_{0}\right)<\tau\right)=\xi$, where $\xi=v([0, \tau])$ in the case of the compound Poisson process and $\xi=1$ in the case of the process with infinite activity of jumps. Using (8), we find that, as $x_{0} \rightarrow 0^{+}$,

$$
\begin{aligned}
\mathrm{P}\left(t_{1}^{*}\left(x_{0}\right)<+\infty, W_{\delta^{*}}+\beta \delta^{*}>0 \mid W_{0}=0\right) & <1-\mathrm{P}\left(W_{\tau}+\beta \tau<0 \mid W_{0}=0\right) \xi \\
& <1-\varepsilon_{2} .
\end{aligned}
$$

To summarize, we have proved that the function

$$
\mathrm{P}\left(x_{0}\right)=\mathrm{P}\left(t_{1}^{*}\left(x_{0}\right)<+\infty, W_{\delta^{*}}+\beta \delta^{*}>0 \mid W_{0}=0\right)
$$

satisfies the following properties:

- for any $c>0$, there exists $\varepsilon_{1}=\varepsilon_{1}(c)>0$ such that $\mathrm{P}\left(x_{0}\right)<1-\varepsilon_{1}(c)$ for all $x_{0}>c$,

- there exists $\varepsilon_{2}>0$ such that $\lim _{x_{0} \rightarrow 0^{+}} \mathrm{P}\left(x_{0}\right)<1-\varepsilon_{2}$.

Therefore, we conclude that there exists $\varepsilon>0$ such that $\mathrm{P}\left(x_{0}\right)<1-\varepsilon$ for all $x_{0} \geq 0$; thus, $C<1-\varepsilon$. This completes the proof in the case where $\beta>0$.

For a complementary point of view, the next result shows that the sequence $\left(t_{i}^{*}\left(x_{0}\right)\right)_{i \geq 1}$ converges pathwise.

Theorem 3. For any time-changed Brownian motion with Lévy subordinator $T_{t}$ and Brownian motion with drift $\beta$, the sequence of stopping times $\left(t_{i}^{*}\left(x_{0}\right)\right)_{i \geq 1}$ converges almost surely to $t^{*}$.

Proof. If $t^{*}=\infty$ then certainly $t_{i}^{*} \rightarrow \infty$, so we suppose that $t^{*}<\infty$. In this case, if $t_{i}^{*}=t_{i+1}^{*}$ for some $i$, the sequence converges, and, thus, the only interesting case to analyze is if $t^{*} \neq t_{i}^{*}$ for all $i<\infty$. Then we have $t_{1}^{*}<t_{2}^{*}<\cdots<t_{i}^{*}<\cdots$. Correspondingly, we have an infinite sequence of excursion overjump intervals which do not overlap: let their endpoints be $T_{i-}^{*}:=T_{t_{i}^{*}-}<T_{i+}^{*}:=T_{t_{i}^{*}}$. The following observations lead to the conclusion.

1. By monotonicity and boundedness of the sequences $\left(T_{i-}^{*}\right)$ and $\left(T_{i+}^{*}\right), \lim _{i \rightarrow \infty} T_{i-}^{*}=$ $\lim _{n \rightarrow \infty} T_{i+}^{*}=T_{\infty}$ exists.

2. $x_{0}+W_{T_{\infty}}+\beta T_{\infty}=0$ by the continuity of Brownian motion.

3. $\lim _{i \rightarrow \infty} t_{i}^{*}=t_{\infty}$ exists and $t_{\infty} \leq t^{*}$.

4. Jump times are totally inaccessible, so there is no time jump at time $t_{\infty}$ almost surely. Hence, $T_{t_{\infty}}=T_{\infty}$.

5. $X_{t_{\infty}}=0$ and so $t_{\infty} \geq t^{*}$; hence, $t_{\infty}=t^{*}$. 


\section{The VG model}

The VG process described in Section 2 is the LSBM where the time-change process $T_{t}$ is the Lévy process with jump measure $\mu(z)=(\nu z)^{-1} \mathrm{e}^{-z / v}$ on $(0, \infty)$ and Laplace exponent $\psi_{T}(u)=(1 / v) \log (1+v u)$. In this section we take $b=0$. This model has been widely used for option pricing, where it has been found to provide a better fit to market data than the Black-Scholes model, while preserving a degree of analytical tractability. The main result in this section reduces the two-dimensional integral representation for $p_{1}^{*}\left(x_{0} ; s, x_{1}\right)$ given in (4) to a one-dimensional integral and leads to greatly simplified numerical computations.

Theorem 4. Define $\alpha=\sqrt{2 / v+\beta^{2}}$. Then

$$
\begin{aligned}
p_{1}^{*}\left(x_{0} ; s, x_{1}\right)= & \frac{\exp \left(\beta\left(x_{1}-x_{0}\right)\right)}{2 \pi v} \int_{\mathbb{R}} \frac{(1+\mathrm{i} v z)^{-s / v}}{\sqrt{\beta^{2}-2 \mathrm{i} z}} \exp \left(-x_{0} \sqrt{\beta^{2}-2 \mathrm{i} z}\right) \\
& \times\left(\exp \left(\left|x_{1}\right| \sqrt{\beta^{2}-2 \mathrm{i} z}\right) \operatorname{Ei}\left(-\left|x_{1}\right|\left(\alpha+\sqrt{\beta^{2}-2 \mathrm{i} z}\right)\right)\right. \\
& \left.\quad-\exp \left(-\left|x_{1}\right| \sqrt{\beta^{2}-2 \mathrm{i} z}\right) \operatorname{Ei}\left(-\left|x_{1}\right|\left(\alpha-\sqrt{\beta^{2}-2 \mathrm{i} z}\right)\right)\right) \mathrm{d} z,
\end{aligned}
$$

where Ei(x) is the exponential integral function (see [11, p. 883]).

Proof. Consider the function $I\left(z_{1}\right)$ which represents the outer integral in (4):

$$
I\left(z_{1}\right)=\frac{1}{2 \pi} \int_{\mathbb{R}} \frac{\log \left(1+\mathrm{i} \nu z_{1}\right)-\log \left(1+\mathrm{i} \nu z_{2}\right)}{\mathrm{i}\left(z_{1}-z_{2}\right)} \frac{\exp \left(-\left|x_{1}\right| \sqrt{\beta^{2}-2 \mathrm{i} z_{2}}\right)}{\sqrt{\beta^{2}-2 \mathrm{i} z_{2}}} \mathrm{~d} z_{2} .
$$

First we perform the change of variables $u=\mathrm{i} \sqrt{\beta^{2}-2 \mathrm{i} z_{2}}$ and obtain

$$
I\left(z_{1}\right)=\frac{1}{\pi} \int_{\mathbb{R}} \frac{\log \left(1+(\nu / 2)\left(u^{2}+\beta^{2}\right)\right)-\log \left(1+\mathrm{i} \nu z_{1}\right)}{u^{2}+\beta^{2}-2 \mathrm{i} z_{1}} \exp \left(\mathrm{i}\left|x_{1}\right| u\right) \mathrm{d} u,
$$

where the contour $L$ obtained from $\mathbb{R}$ under the map $z_{2} \rightarrow u=\mathrm{i} \sqrt{\beta^{2}-2 \mathrm{i} z_{2}}$ is transformed into the contour $\mathbb{R}$ (this is justified since the integrand is an analytic function in this region for any $z_{1}$ ). To complete the proof, we separate the logarithms, i.e.

$$
\log \left(1+\frac{v}{2}\left(u^{2}+\beta^{2}\right)\right)-\log \left(1+\mathrm{i} v z_{1}\right)=\log (u+\mathrm{i} \alpha)+\log (u-\mathrm{i} \alpha)-\log \left(\frac{2}{v}+2 \mathrm{i} z_{1}\right),
$$

and use the partial fractions decomposition

$$
\frac{1}{u^{2}+\beta^{2}-2 \mathrm{i} z_{1}}=\frac{1}{2 \mathrm{i} \sqrt{\beta^{2}-2 \mathrm{i} z_{1}}}\left(\frac{1}{u-\mathrm{i} \sqrt{\beta^{2}-2 \mathrm{i} z_{1}}}-\frac{1}{u+\mathrm{i} \sqrt{\beta^{2}-2 \mathrm{i} z_{1}}}\right)
$$

to obtain six integrals, which can be computed by shifting the contours of integration and using the following Fourier transform formulae (see [11, Formulae 6.232, p. 639]):

$$
\begin{array}{ll}
\int_{\mathrm{i} \varepsilon+\mathbb{R}} \log \left(1+\frac{\mathrm{i} y}{b}\right) \mathrm{e}^{\mathrm{i} x y} \frac{\mathrm{d} y}{y}=-2 \pi \mathrm{i} E i(-b x), & b>0, \\
\int_{\mathrm{i}(b+\varepsilon)+\mathbb{R}} \log \left(\frac{\mathrm{i} y}{b}-1\right) \mathrm{e}^{\mathrm{i} x y} \frac{\mathrm{d} y}{y}=-2 \pi \mathrm{i} E i(b x), & b>0 .
\end{array}
$$


Remark. Using the change of variables $u=\mathrm{i} \sqrt{\beta^{2}-2 \mathrm{i} z}$ and simplifying the expression, we can obtain a simpler formula for $p_{1}^{*}\left(x_{0} ; s, x_{1}\right)$ :

$$
\begin{gathered}
p_{1}^{*}\left(x_{0} ; s, x_{1}\right)=\exp \left(\beta\left(x_{1}-x_{0}\right)\right) \frac{(v / 2)^{-s / v-1}}{2 \pi \mathrm{i}} \int_{\mathbb{R}}\left(\alpha^{2}+y^{2}\right)^{-s / v} \sin \left(x_{0} y\right) \exp \left(\mathrm{i}\left|x_{1}\right| y\right) \\
\times E i\left(-\left|x_{1}\right|(\alpha+\mathrm{i} y)\right) \mathrm{d} y .
\end{gathered}
$$

Applying the Plancherel formula to the above expression gives us the following representation for $p_{1}^{*}$ :

$$
\begin{aligned}
p_{1}^{*}\left(x_{0} ; s, x_{1}\right)= & \exp \left(\beta\left(x_{1}-x_{0}\right)-\alpha\left(x_{0}+\left|x_{1}\right|\right)\right) \frac{\left(v \alpha^{2} / 2\right)^{-s / v}}{\sqrt{\pi} v\left(x_{0}+\left|x_{1}\right|\right)} \frac{\Gamma(s / v+1 / 2)}{\Gamma(s / v+1)} \\
+ & \sqrt{\frac{2 \alpha^{3}}{\pi}} \exp \left(\beta\left(x_{1}-x_{0}\right)-\alpha\left|x_{1}\right|\right) \\
& \times \frac{(\alpha \nu)^{-s / v-1}}{\Gamma(s / v)} \int_{0}^{\infty} u^{s / v-1 / 2} K_{s / v-1 / 2}(\alpha u) f\left(x_{0}, x_{1} ; u\right) \mathrm{d} u
\end{aligned}
$$

where

$$
f\left(x_{0}, x_{1} ; u\right)=\frac{\exp \left(-\alpha\left(u+x_{0}\right)\right)}{u+x_{0}+\left|x_{1}\right|}-\operatorname{sgn}\left(u-x_{0}\right) \frac{\exp \left(-\alpha\left|u-x_{0}\right|\right)}{\left|u-x_{0}\right|+\left|x_{1}\right|}-2 \frac{\exp \left(-\alpha\left(u+x_{0}\right)\right)}{x_{0}+\left|x_{1}\right|} .
$$

The above expression is useful for computations when $s$ is small. In particular, when $s=0$, we find that

$$
p_{1}^{*}\left(x_{0} ; 0, x_{1}\right)=\frac{\exp \left(\beta\left(x_{1}-x_{0}\right)-\alpha\left(x_{0}+\left|x_{1}\right|\right)\right)}{v\left(x_{0}+\left|x_{1}\right|\right)} .
$$

\section{Numerical implementation for the VG model}

The algorithm for computing the functions $p^{*}\left(x_{0} ; s, x\right)$ and $p^{*}\left(x_{0} ; s\right)$ can be summarized as follows.

1. Choose the discretization step sizes $\delta_{x}$ and $\delta_{t}$ and the discretization intervals $[-X, X]$ and $[0, T]$. The grid points are $t_{i}=i \delta_{t}, 1 \leq i \leq N_{s}$, and $x_{j}=\left(j+\frac{1}{2}\right) \delta_{x},-N_{x} \leq j \leq N_{x}$.

2. Compute the three-dimensional array $p_{1}^{*}\left(x_{i} ; t_{j}, x_{k}\right)$. For $j>0$, use (9) and, for $j=0$, use explicit formula (10).

3. Iterate (2) or (3). This step can be considerably accelerated if the convolution in the $u$-variable is done using FFT methods. We used the midpoint rule for integration in the $y$ - and $u$-variables.

Theorem 2 implies that step 3 in the above algorithm has to be repeated only a few times. In practice, we found that 3-4 iterations were usually enough. An important empirical fact is that the above algorithm works quite well with just a few discretization points in the $x$-variable. We found that if we used a nonlinear grid (which places more points $x_{i}$ near $x=0$ ) then the above algorithm produced reasonable results with values of $N_{x}$ as small as 10 or 20 .

We compared our algorithm for the PDF $p^{*}\left(x_{0} ; s\right)$ to a finite-difference method that was implemented as follows. First we approximated the first passage time by its discrete counterpart:

$$
\hat{t}^{*}=\hat{t}^{*}(x)=\min \left\{t_{i}: X_{t_{i}}<0 \mid X_{0}=x\right\},
$$



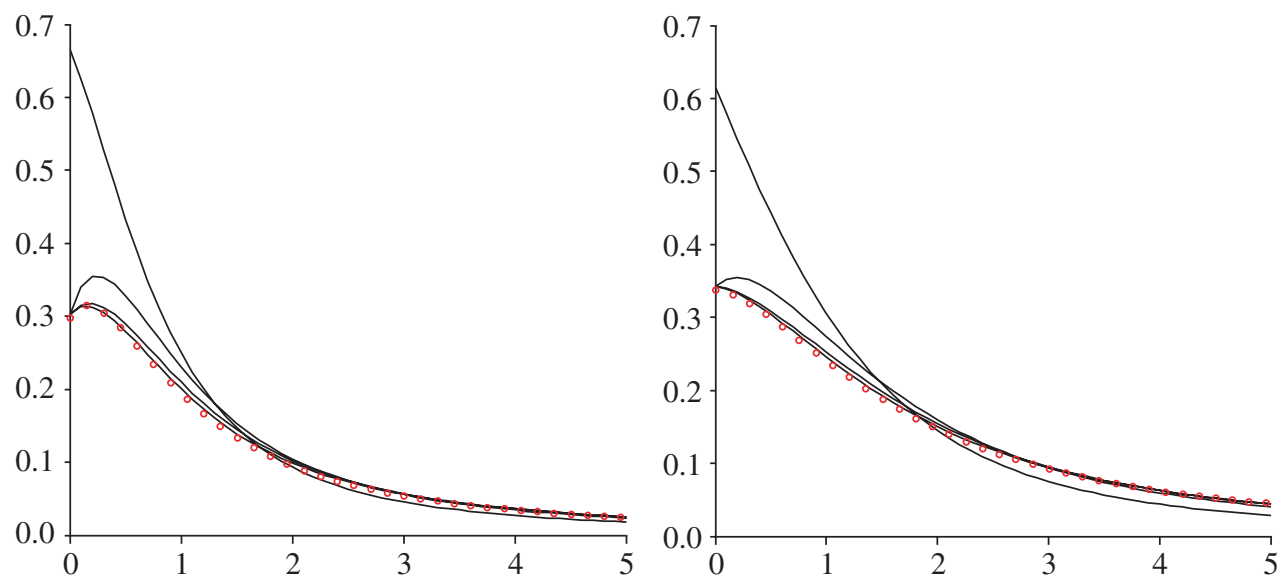

Figure 2: The density of the first passage time for the two sets of parameters. The circles show the 'exact' result and the three solid lines show the first three approximations.

where $t_{i}=i \delta_{t}, 0 \leq i \leq n_{t}$, is the discretization of the interval [0,T]. The probabilities $f_{i}(x)=\mathrm{P}\left(\hat{t}^{*}>t_{i} \mid X_{0}=x\right)$ satisfy the iteration

$$
f_{i+1}(x)=\mathbf{1}_{\{x>0\}} \int_{\mathbb{R}} p\left(\delta_{t}, x-y\right) f_{i}(y) \mathrm{d} y, \quad i \geq 1,
$$

with $f_{0}(x)=\mathbf{1}_{\{x>0\}}$, and can be computed numerically with the following steps.

1. Discretize the space variables $x=i \delta_{x}$ and $y=j \delta_{x}, 0<i, j<n_{x}$.

2. Compute the array of transitional probabilities $\hat{p}_{i}=p\left(\delta_{t}, x_{i}\right)$, and normalize $\hat{p}_{0}$ so that $\sum_{i} \hat{p}_{i}=1$.

3. Use the convolution (based on the FFT) to iterate (11) $n_{t}$ times.

4. Compute the approximation of the first passage time density

$$
\hat{p}^{*}\left(x, t_{i}+\frac{\delta_{t}}{2}\right)=\frac{f_{i+1}(x)-f_{i}(x)}{\delta_{t}} .
$$

The big advantage of this method is that it is explicit and unconditionally stable: we can choose the number of discretization points in $x$-space and $t$-space independently. This is not true in general explicit finite-difference methods, where we would solve the Fokker-Planck equation by discretizing the Markov generator and the time derivative, since $\delta_{t}$ and $\delta_{x}$ have to lie in a certain subset in order for the methods to be stable.

Figure 2 summarizes the numerical results for the PDF $p^{*}\left(x_{0} ; s\right)$ over the time interval $[0,5]$ for the VG model with the following two sets of parameters.

Set I. $x_{0}=0.5, \beta=0.2$, and $v=1$.

Set II. $x_{0}=0.5, \beta=-0.2$, and $v=2$.

The number of grid points used was $N_{t}=50$ and $N_{x}=10$. The circles correspond to the solution obtained by a high-resolution finite-difference PIDE method as described above 

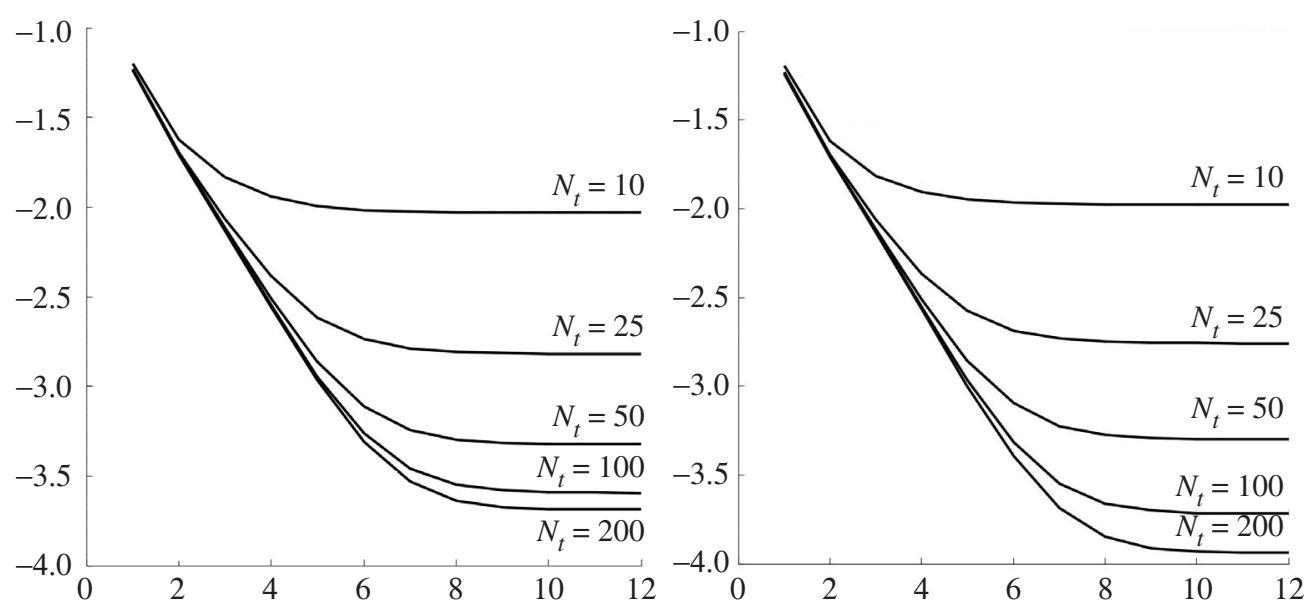

FIGURE 3: The error, $\log _{10}\left(\left\|p^{*}-p_{i}^{*}\right\|_{L_{1}}\right)$, for the new approach plotted against the number of iterations. The left-hand plot has $N_{x}=10$, the right-hand plot has $N_{x}=20$, and $N_{t} \in\{10,25,50,100,200\}$.

TABLE 1: Computation time (seconds) for the new approach.

\begin{tabular}{lcccccc}
\hline & $N_{x}$ & \multicolumn{5}{c}{$N_{t}$} \\
\cline { 3 - 7 } & & 10 & 25 & 50 & 100 & 200 \\
\hline Precomputing time & 10 & 0.0313 & 0.0259 & 0.0324 & 0.0461 & 0.0687 \\
Each iteration & & 0.0006 & 0.0008 & 0.0011 & 0.0021 & 0.0046 \\
\hline Precomputing time & 20 & 0.0645 & 0.0612 & 0.0745 & 0.0868 & 0.1298 \\
Each iteration & & 0.0037 & 0.0045 & 0.0066 & 0.0120 & 0.0269 \\
\hline
\end{tabular}

(with $n_{t}=1000$ and $n_{x}=10000$ ), and the black lines show successive iterations $p_{i}^{*}\left(x_{0}, t\right)$ converging to $p^{*}\left(x_{0}, t\right)$. As we see, three iterations of (3) provide a visually acceptable accuracy in a running time of less than 0.1 seconds (on a $2.5 \mathrm{GHz}$ laptop).

Figure 3 illustrates the convergence of our method and Table 1 shows the computation times (on the same $2.5 \mathrm{GHz}$ laptop). We used the parameters of set II for the VG process, and the PIDE method with $n_{t}=1000$ and $n_{x}=10000$ to compute the 'exact' solution $p^{*}\left(x_{0}, t\right)$. Figure 3 shows the $\log _{10}$ of the error

$$
\left\|p^{*}-p_{i}^{*}\right\|_{L_{1}}=\int_{0}^{T}\left|p^{*}\left(x_{0}, t\right)-p_{i}^{*}\left(x_{0}, t\right)\right| \mathrm{d} t
$$

on the vertical axis and the number of iterations on the horizontal axis; different curves correspond to a different number of discretization points in $t$-space. The number of discretization points in $x$-space is fixed at $N_{x}=10$ for the left-hand plot and $N_{x}=20$ for the right-hand plot. We see that initially the error decreases exponentially and then flattens out. The flattening indicates that our method converges to the wrong target (which is to be expected since there is always a discretization error coming from $N_{x}$ and $N_{t}$ being finite). However, increasing $N_{t}$ and $N_{x}$ brings us closer to the 'target'. In Table 1 we show the precomputing time needed 


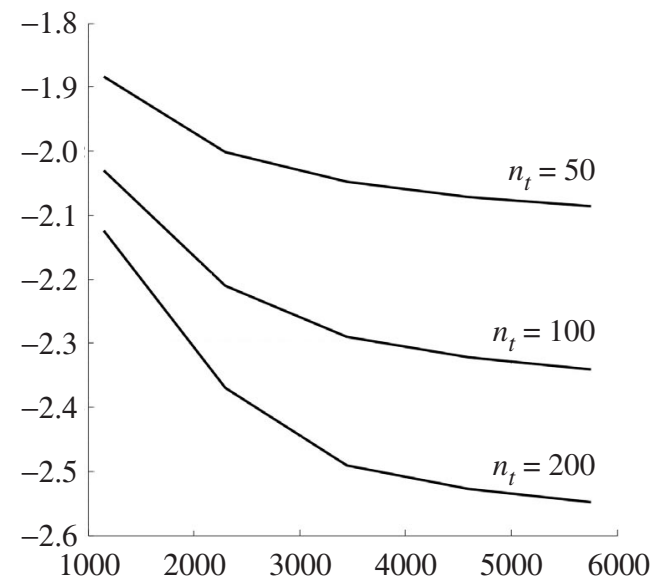

FIGURE 4: The error, $\log _{10}\left(\left\|p^{*}-\hat{p}^{*}\right\|_{L_{1}}\right)$, for the finite-difference method is plotted against the number of grid points $n_{x}$ for the values $n_{t}=50,100$, and 200 .

TABLE 2: Computation time (seconds) for the finite-difference approach.

\begin{tabular}{rccccc}
\hline \multirow{2}{*}{$N_{t}$} & \multicolumn{5}{c}{$n_{x}$} \\
\cline { 2 - 6 } & 1150 & 2300 & 3450 & 4600 & 5750 \\
\hline 50 & 0.0756 & 0.2935 & 0.9582 & 1.8757 & 2.9967 \\
100 & 0.1456 & 0.5821 & 1.9397 & 3.7409 & 6.0026 \\
200 & 0.2870 & 1.1478 & 3.8833 & 7.4768 & 11.9935 \\
\hline
\end{tabular}

to compute the three-dimensional array $p_{1}^{*}\left(x_{i} ; t_{j}, x_{k}\right)$ and the time needed to perform each iteration (3).

To put these results into perspective, Figure 4 and Table 2 show similar results for the finitedifference method. In Figure 4 we show the same logarithm of the error on the vertical axis and the number of discretization points $n_{x}$ on the horizontal axis. Different curves correspond to $n_{t} \in\{50,100,200\}$. The running time presented in Table 2 includes only the time needed to perform $n_{t}$ convolutions (11) using the FFT. As we can see by comparing Figures 3 and 4, even with a relatively large number of discretization points, $n_{x}=5750$ and $n_{t}=200$, the accuracy produced by a finite-difference method is an order of magnitude worse than the accuracy produced by our method (with much fewer discretization points). Moreover, we can see that the running times of the PIDE method are consistently orders of magnitude larger.

\section{Conclusions}

First passage times are an important modeling tool in finance and other areas of applied mathematics. The main result of this paper is the theoretical connection between two distinct notions of first passage time that arise for LSBMs. This relation leads to a new way to compute true first passage for these processes that is apparently less expensive than finite-difference methods for a given level of accuracy. Our paper opens up many avenues for further theoretical 
and numerical work. For example, the methods we describe are certainly applicable for a much broader class of time-changed Brownian motions and time-changed diffusions. Finally, it will be worthwhile to explore the use of the first passage of the second kind as a modeling alternative to the usual first passage time.

\section{Acknowledgement}

The authors would like to thank Professor Martin Barlow for providing the proof of Theorem 4 .

\section{References}

[1] Alili, L and Kyprianou, A. E. (2005). Some remarks on first passage of Lévy processes. Ann. Appl. Prob. 15, 2062-2080.

[2] Applebaum, D. (2004). Lévy Processes and Stochastic Calculus (Camb. Stud. Adv. Math. 93). Cambridge University Press.

[3] Asmussen, S. (2000). Ruin Probabilities (Adv. Ser. Statist. Appl. Prob. 2). World Scientific, River Edge, NJ.

[4] Asmussen, S., Avram, F. and Pistorius, M. R. (2004). Russian and American put options under exponential phase-type Lévy models. Stoch. Process. Appl. 109, 79-111.

[5] Barndorff-Nielsen, O. E. (1997). Normal inverse Gaussian distribution and stochastic volatility modelling. Scand. J. Statist. 24, 1-13.

[6] Bertoin, J. (1996). Lévy Processes (Camb. Tracts Math. 121). Cambridge University Press.

[7] Borodin, A. N and Salminen, P. (1996). Handbook of Brownian Motion-Facts and Formulae. Birkhäuser, Basel.

[8] Cherny, A. S and Shiryaev, A. N. (2002). Change of time and measures for Lévy processes. (Lectures from the Summer School 'From Lévy processes to semimartingales: recent theoretical developments and applications to finance' (Aarhus, August 2002)).

[9] Cont, R. and Tankov, P. (2004). Financial Modeling with Jump Processes. Chapman \& Hall, Boca Raton, FL.

[10] Cont, R. and Voltchkova, E. (2005). A finite difference scheme for option pricing in jump diffusion and exponential Lévy models. SIAM J. Numer. Anal. 43, 1596-1626.

[11] Gradshteyn, I. S. AND Ryzhik, I. M. (2000). Tables of Integrals, Series, and Products, 6th edn. Academic Press, San Diego, CA.

[12] Hurd, T. R. (2007). Credit risk modelling using time-changed Brownian motion. Working paper. Available at http://www.math.mcmaster.ca/tom/HurdTCBMRevised.pdf.

[13] Jacod, J. AND Shiryaev, A. N. (1987). Limit Theorems for Stochastic Processes. Springer, Berlin.

[14] Kou, S. G And Wang, H. (2003). First passage times of a jump diffusion process. Adv. Appl. Prob. 35, 504-531.

[15] Kyprianou, A. E. (2006). Introductory Lectures on Fluctuations of Lévy Processes with Applications. Springer, Berlin.

[16] LewIS, A. L. AND MordecKI, E. (2008). Wiener-Hopf factorization for Lévy processes having positive jumps with rational transforms. J. Appl. Prob. 45, 118-134.

[17] Madan, D. and Seneta, E. (1990). The VG model for share market returns. J. Business 63, 511-524.

[18] MoRdECKI, E. (2002). The distribution of the maximum of a Lévy process with positive jumps of phase-type. Theory Stoch. Process. 8, 309-316.

[19] O’Cinneide, C. A. (1990). Characterization of phase-type distributions. Stoch. Models 6, 1-57.

[20] PercheskiI, E. A. And Rogozin, B. A. (1969). On the joint distribution of random variables associated with fluctuations of a process with independent increments. Theory Prob. Appl. 14, 410-423.

[21] Rogers, L. C. G. (1984). A new identity for real Lévy processes. Ann. Inst. H. Poincaré Prob. Statist. 20, 21-34. 